\title{
Family Reliance on Physicians' Decisions in Life-Sustaining Treatments in Acute-on-Chronic Respiratory Diseases in a Respiratory ICU: A Single-Center Study
}

\author{
Filipe Monteiro MD
}

\begin{abstract}
BACKGROUND: In ICUs, many patients are unable to participate in decision-making regarding life-sustaining treatments. This study evaluated the opinions of family members about family and physician participation in life-sustaining treatment decisions and examined factors that influence those decisions. METHODS: This was a prospective exploratory observational study that used convenience sampling. Inquiry interviews were conducted over a 3-year period, with 126 family members (out of 303 potential participants) of patients with acute-on-chronic respiratory failure, who had been admitted to the respiratory ICU and were dependent on invasive or noninvasive mechanical ventilation. Patients of $\leq 18$ years old, with a stay of $<3$ days, and oncologic patients were excluded. RESULTS: Ninety-eight percent $(123 / 126)$ of the participant family members had an opinion about their involvement in decision-making about life-sustaining treatments. Physician choice was preferred by $54 / 123(44 \%), 55 / 123(45 \%)$ wished to share the decision with the physician, and $14 / 123(11 \%)$ wished the family to decide. All the patients were incompetent at the time of inquiry. Autonomy prior to admission to the respiratory ICU influenced the decision. CONCLUSIONS: A majority of the families relied on physicians to help in the decision-making process about life-sustaining treatments in patients with acute-on-chronic respiratory diseases. From the family's point of view, the principle of autonomy can be exercised by delegating the decision-making process to the physician. To assume a uniform ethical conduct is to antagonize the definition of ethics. Key words: end of life; surrogate; decision-making; death. [Respir Care 2014;59(3):411-419. (c) 2014 Daedalus Enterprises]
\end{abstract}

\section{Introduction}

ICUs were originally conceived for acute conditionsillness or injuries. Subsequently their scope was extended to acute-on-chronic diseases, and today ICUs often admit patients with end-stage diseases and high mortality risk. ${ }^{1}$ This trend is expected to have important implications regarding patient involvement in end-of-life treatment deci-

The author is affiliated with Pulmonology Department 1, Santa Maria University Hospital, Lisbon, Portugal.

The author has disclosed no conflicts of interest.

Correspondence: Filipe Monteiro MD, Pulmonology Department 1, Santa Maria University Hospital, Centro Hospitalar Lisboa Norte, Avenida Professor Egas Moniz, 1649-028 Lisbon, Portugal. E-mail: jfpmonteiro@ yahoo.com.

DOI: $10.4187 /$ respcare.02695 sions, mainly through advance directives. In southern European countries, such as Portugal, where advance directives are uncommon, the first steps are being taken through discussion and legislation. However, despite their utility, only $20-30 \%$ of patients have an advance directive, even in Anglo-Saxon countries, where advance directives are encouraged. ${ }^{2}$

Thus, if the patient is incompetent to decide, ${ }^{3}$ because of an acute condition or heavy sedation, and has no valid advance directives regarding life-sustaining treatments, the decision-making shifts from the patient to the family. The legitimacy of family members as decision makers derives from the close relationship that normally exists among family members or, as Dan Brock expresses it, "patientregarding grounds for family authority." 4

Serious concerns exist, however, about the philosophical justification for substituted judgment. ${ }^{5}$ In addition, the practice of the principle of autonomy is not uniformly understood and exerted in all countries, as is shown in the 
differences between Anglo-Saxon and southern European countries, ${ }^{6}$ which have different attitudes about end-of-life matters. ${ }^{7-9}$ As one example, decision-making is predominantly physician-centered in Europe, whereas in the United States the patients and families are much more involved in the process. ${ }^{10}$ Most ICU patients are elderly, so the responsibility for decision-making most frequently falls on younger people. In Europe, people in different countries hold widely varying views about their intergenerational responsibilities to their elders. For example, in Spain, Italy, Greece, and Portugal, people feel strongly bound by obligations toward their parents, whereas in Finland, Ireland, and the Netherlands, children's obligations to parents are thought to be minimal. ${ }^{11}$ Hence, it is important to know the family member variables that can influence the decision.

The present study was conducted in a single respiratory ICU in a university hospital in Lisbon, Portugal, where patients' families are seldom requested to participate directly in the decision-making process. ${ }^{12}$ The aim was to understand family participation in decisions about end-oflife issues in the respiratory ICU. Do family members wish to help decide about life-sustaining treatments for patients who are incompetent to decide? Would they rather delegate such matters to the physician? What variables influence that choice?

The factors that can influence the decision-making process are, on the one hand, related to the patient (age, comorbidities, independence in daily-life activities before admission, and competency at the time of inquiry), and, on the other hand, to the family members, both demographically (age, sex, occupation, co-habitation) and nondemographically (relationship with patient, depression, prognosis). ${ }^{6}$

Another important factor is that end-of-life decisions are not solely rational; they include cultural and emotional components. ${ }^{13}$ In our study we considered the relevance of depression, ${ }^{14}$ communication (because it is well known that patients and families who trust their physicians feel comfortable relying on the physician for help in making decisions ${ }^{15}$ ), and prognostic implications, ${ }^{16}$ since, despite prognostic uncertainty, they allow families to make a decision based on the likely outcomes of treatments. We therefore studied the perceptions of family members and factors that influence the decision-making process concerning life-sustaining treatments.

\section{Methods}

This prospective exploratory observational study was approved by the ethics committee of Santa Maria Hospital and authorized by the Portuguese National Committee for Data Protection. The study design, procedure, and results

\section{QUICK LOOK}

\section{Current knowledge}

When patients with acute-on-chronic respiratory failure requiring mechanical ventilation are unable to participate in end-of-life decisions, family members are asked to make these difficult decisions.

\section{What this paper contributes to our knowledge}

In Portugal a majority of surveyed family members relied on physicians to help in the decision-making process. From the family members' point of view, the principle of autonomy can be exercised by surrogating the decision-making to the physician. The sociological and cultural characteristics of individual countries should be considered in these decisions.

were monitored by an independent statistical consultancy agency.

This study was conducted over 3 years (October 2007 to March 2011) in the respiratory ICU of the Pulmonology Department of Santa Maria University Hospital, a 14-bed unit (8 high-risk and 6 intermediate noninvasive ventilation beds), in Lisbon, Portugal. The unit has 3 consultant physicians and 2 assistant physicians. We interviewed a convenience sample (ie, available and willing to participate) of family members of patients in the respiratory ICU who had acute-on-chronic respiratory failure and were dependent on invasive or noninvasive mechanical ventilation. We excluded patients $\leq 18$ years old, whose ICU stay was $<3$ days, and whose pathology was oncologic.

For each patient we recorded ICU stay, diagnosis, type of ventilation, age, sex, religion, household, occupation, acute physiology score and Acute Physiology and Chronic Health Evaluation (APACHE II) score, previous health state, previous episodes of mechanical ventilation, and advance directives (if any). For each family member we recorded age, sex, relationship to the patient, cohabitation, residency, education, occupation, religion, communication with the staff doctor, depression according to the Hamilton Depression Rating Scale, the family member's rating of the ICU care (very good, good, reasonable, or bad), and the family member's understanding of the patient's prognosis (very good, good, reasonable, or bad).

Before they answered the questions, each family member was informed about the severity of the disease, on a scale of 1-10 (considering the APACHE II severity index) and assured that answers would not be considered in any decisions regarding life-sustaining treatments.

A physician administered the questionnaire, first taking some time in conversation to put the family at ease and to establish an informal atmosphere. The administering phy- 


\section{Family Reliance on Physicians’ Decisions in Life-Sustaining Treatments}

sician recorded the answers after making sure of their content and meaning. Each family member was asked the following question: Considering that your next of kin is incompetent, in case of life-sustaining treatments, who should be responsible for that decision? The following options were given to the family members.

- Family

- Physician

- Family plus physician

- Don't know or no answer

The competency of the patient depended on their mental abilities and cognitive capabilities required to rationally execute a legally recognized decision. ${ }^{17}$

\section{Statistical Analysis}

The results were analyzed with descriptive univariable and bivariable statistics, using average, standard deviation, median, and quartiles for the quantitative variables, and counts and percentages for the qualitative variables. The significance of the association between 2 qualitative variables was assessed using the chi-square independence test or the Fisher exact test where appropriate. All tests were 2-sided, using a type- 1 error of $5 \%$ (unless otherwise stated). All statistical analyses were made with statistics software (SPSS 19, SPSS, Chicago, Illinois).

\section{Results}

One visiting family member per patient participated in the inquiry. There were 126 (42\%) interviewees out of 303 potential participants.

\section{Univariable Exploratory Analysis}

Patient Statistics. The majority of patients were elderly, with an average age of approximately 71 years and a median of 74 years. About $41 \%$ were $>75$ years old, ${ }^{18}$ and $29 \%$ were $\leq 65$ years old. Males accounted for $65 \%(n=82)$ of the patients, and $94(75 \%)$ lived with family members. Their educational level was very low: $59 \%$ had very low or low literacy (according to the analphabets/IV standard of education). There were 119 (94\%) whites, and $113(90 \%)$ were retired.

Acute-on-chronic respiratory failure from chronic respiratory disease, with or without congestive heart failure, was responsible for $102(81 \%)$ of ICU admissions. The median Acute Physiology Score and APACHE II scores were 17 and 26, respectively (upper limits 35 and 45). Invasive mechanical ventilation was required by 71 patients $(56 \%)$.
In terms of previous health, 99 (79\%) had not previously required mechanical ventilation. Most of the patients, 114 (91\%), had no advance directives regarding mechanical ventilation, and 112 (89\%) had none regarding hemodialysis. All the expressed directives were verbal, not written. A large number of patients, 113 (90\%), had been lucid prior to their hospitalization (ie, could think clearly and rationally), and the exacerbation was probably responsible for the incompetency. Further, before respiratory ICU admission, 77 (61\%) were physically autonomous (independent in activities of daily living).

Family Member Statistics. The average age was 53 years, and the median was 55 years. The family visitors were mostly sons, daughters, and in-laws. Because females are more typically frequent visitors in Portugal, 97 (77\%) were female. A close relationship between the patient and visitor (spouse, adult son/daughter, parent or sibling) existed in 105 (83\%) of the participants, so in most cases the relationship was close enough for the participant to know the patient's values and expectations.

Despite close family ties, 90\% (113) lived in urban areas and did not live with the patient. Secondary or university education accounted for $47 \%$. The difference in literacy compared to the patients can be explained by the age of the family members and the economic and social evolution of the country in recent decades. In terms of employment, 71 (56\%) were employed, and 111 (88\%) considered themselves religious. The majority of family members (66\%) had some degree of depression (slight, moderate, or serious), as measured by the Hamilton Depression Rating Scale. No anti-depression treatment was prescribed to those who had depression.

All who participated were asked about the medical information provided to them. The majority 88 (70\%) were interested in it, but many desired different information: 56 (44\%) wanted a general explanation, and $58(46 \%)$ wanted a more specific elucidation. Even so, 68 (54\%) considered themselves enlightened, and 58 (46\%) felt more comfortable after receiving information from the physician. A majority, 99 (79\%), classified the care provided in the ICU as very good. Fifty-eight (46\%) family members believed that the prognosis of their patient was "bad." The global mortality was $60 \%$.

\section{Bivariable Exploratory Analysis}

We established 3 categories of family members: those who favored a physician decision on life-saving treatment; those who preferred a shared decision-making; and those who preferred the decision be solely from the family.

Fifty-four family members opted for physician decision, 55 for shared decision, and 14 for family decision. One- 


\section{Family Reliance on Physicians’ Decisions in Life-Sustaining Treatments}

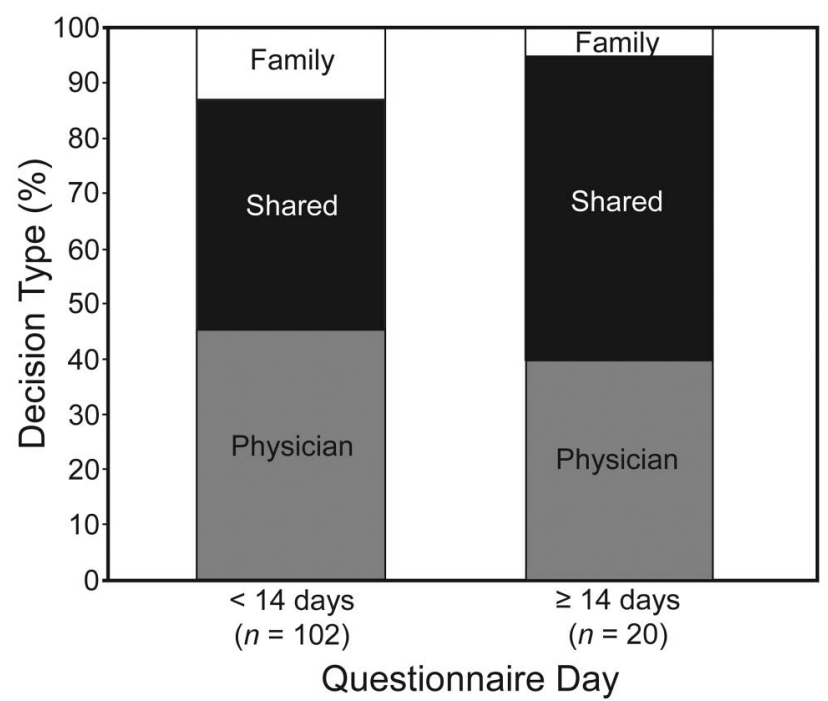

Fig. 1. Effect of duration of mechanical ventilation on family members' preferences about decision-making about life-saving treatments. We chose 14 days as representing prolonged ventilation.

hundred nine family members preferred physician involvement (ie, preferred either physician decision or shared decision-making), whereas 17 preferred no physician involvement (family decision) $(P<.001)$. However, the small number that preferred family decision-making may introduce bias in the analysis.

We also analyzed if the number of mechanical-ventilation days at the time of inquiry had any relation to the family members' preference. In general, from a clinical point of view, ventilation is considered prolonged at $\geq 21$ days. ${ }^{19}$ However, since in this study the evaluation of prolonged ventilation was not from a clinical perspective but from the family's emotional perspective, we subjectively chose $\geq 14$ days as the cutoff, which is a period of time that can have some influence in the options of decision-making. Thus, considering the 2 groups (Fig. 1), it was verified that with a stay $\geq 14$ days the family members' preference tended to shift toward shared decisionmaking. But, again, the small number of patients in the prolonged-ventilation group may introduce bias in the analysis.

Tables 1, 2, and 3 show the results of the influence of some of the variables on the families' preferences.

When the patient's previous health state (lucidity and autonomy in activities of daily living before ICU admission) was considered (see Table 1), because a great majority of patients in all 3 groups (physician decision, shared decision, and family decision) had been lucid, there was no difference between physician and family participation. In the case of autonomous patients prior to admission, family members leaned strongly toward a physician decision, whereas a family decision was favored for patients who were completely dependent before admission in the ICU.

Invasive mechanical ventilation can be a traumatic experience for both patient and family. In acute-on-chronic respiratory diseases, patients are likely to undergo repeated invasive mechanical ventilation. ${ }^{20}$ Thus, this variable should be considered in analyzing the family's preferences. In the present study, although the number of patients who had previously undergone mechanical ventilation was small (27), a majority of family members with previous experience did not want physician involvement. Had they any fear of medical futility? In an opposite option, family members having no previous experience with invasive mechanical ventilation favored physician involvement, choosing either physician-only decision-making (76\%) or shared decision-making (84\%).

Advance directives regarding mechanical ventilation $(12 / 126,10 \%)$, hemodialysis $(14 / 126,11 \%)$, or cardiopulmonary resuscitation $(13 / 126,10 \%)$ were all verbal. In Portugal there is no data regarding advance directives, because the relevant legal implementation is very recent. In terms of the main diagnosis, the families of patients with congestive heart failure without any chronic respiratory disease chose physician decision $(16 / 17$, alone or shared). Regarding the mode of ventilation, for invasive mechanical ventilation, 63/71 families chose to entrust the physician with the decision (alone or shared). The severity of the situation (intubation, ventilation, or sedation) from the family perspective shifted the decision toward the physician.

In the patient demographics analysis it was verified that family members of older patients prefer a physician decision (a 75-year median compared to 72 for shared and 70 without the physician). However, the $P$ value was not significant $(P=.83)$. In terms of cohabitation, $89 \%$ who opted for total physician responsibility, $60 \%$ of those who chose shared decision, and $86 \%$ of those choosing no physician partnership live with the patient $(P=.18)$. Thus, it seems that when family members live with the patient they are more likely to take the responsibility for decisionmaking. The proximity resulting from cohabitation leads to a better knowledge of the patient's values, so the family member is more liable to assume involvement in the decision-making process.

Although the education level of the patients was generally quite low in all the groups, the family members of patients with more education chose shared decisionmaking $(29 \%)$ more than physician decision-making $(11 \%)$ or family decision-making $(14 \%)$. A higher literacy level of the patient could demonstrate an intrinsic desire of his family member to know and dialogue with the physician about the disease $(P=.56)$. A different behavior was seen with regard to the education level of the family member (see Table 2). Those with a second- 


\section{Family Reliance on Physicians' Decisions in Life-Sustaining Treatments}

Table 1. Patient Health State and Advance Directives Before Admission

\begin{tabular}{|c|c|c|c|c|c|}
\hline & \multicolumn{4}{|c|}{ Family Member's Preference About Life-Saving Treatment Decisions, \% } & \multirow[b]{2}{*}{$P$} \\
\hline & $\begin{array}{l}\text { Physician } \\
\text { Decision } \\
(n=54)\end{array}$ & $\begin{array}{c}\text { Shared } \\
\text { Decision } \\
(n=55)\end{array}$ & $\begin{array}{c}\text { Family } \\
\text { Decision } \\
(n=14)\end{array}$ & $\begin{array}{c}\text { Don't Know/ } \\
\text { Didn't Answer } \\
(n=3)\end{array}$ & \\
\hline \multicolumn{6}{|l|}{ Lucidity } \\
\hline Lucid & 92 & 86 & 93 & 100 & \\
\hline Partially lucid & 6 & 7 & 7 & 0 & \\
\hline Confused & 2 & 7 & 0 & 0 & .43 \\
\hline \multicolumn{6}{|l|}{ Autonomy } \\
\hline Total autonomy & 70 & 55 & 43 & 100 & \\
\hline Partial autonomy & 18 & 36 & 21 & 0 & \\
\hline Totally dependent & 6 & 9 & 36 & 0 & \\
\hline Bed-ridden & 6 & 0 & 0 & 0 & .09 \\
\hline \multicolumn{6}{|l|}{ Mechanical ventilation } \\
\hline Yes & 24 & 16 & 36 & 0 & \\
\hline No & 76 & 84 & 64 & 100 & .26 \\
\hline \multicolumn{6}{|c|}{ Advance directive instructions } \\
\hline \multicolumn{6}{|c|}{ Mechanical ventilation } \\
\hline Yes & 4 & 4 & 0 & 0 & \\
\hline No & 9 & 4 & 7 & 0 & \\
\hline Unknown & 87 & 92 & 93 & 100 & .76 \\
\hline \multicolumn{6}{|l|}{ Hemodialysis } \\
\hline Yes & 9 & 2 & 7 & 0 & \\
\hline No & 7 & 4 & 7 & 0 & \\
\hline Unknown & 84 & 94 & 86 & 100 & .29 \\
\hline \multicolumn{6}{|c|}{ Cardiopulmonary resuscitation } \\
\hline Yes & 9 & 0 & 4 & 0 & \\
\hline No & 7 & 6 & 4 & 0 & \\
\hline Unknown & 84 & 94 & 92 & 100 & .15 \\
\hline
\end{tabular}

ary or university level education chose family decisionmaking $(57 \%)$, while those with low or very low literacy opted for physician decision-making (37\%). The previous reasoning in the situation of literacy level favors the preference for family autonomy in the decisionmaking process $(P=.56)$.

Although the majority of family members in the 3 groups considered themselves religious, the proportion with religion was higher $(94 \%)$ in the physician-decision group than in the family-decision or shared-decision group, both of which had values at or below $86 \%(P=.12)$. When depression was considered, there was a trend toward physician decision-making (alone or shared) according to the severity of the depression, but the association was not statistically significant $(P=.52)$ (see Table 3$)$.

The family's satisfaction with the care provided seemed to have no influence on their preferences. Regarding the family's understanding of the prognosis, 2 groups were considered: very good/satisfactory and bad. There was a preference for physician involvement (alone or shared) in both groups, although the relationship was not statistically significant $(P=.83)$.

\section{Inferential Analysis}

For the inferential data analysis, the end points (physician decision, shared decision, and family decision) were crossed with:

- Demographics (sex, age, education, cohabitation, employment status, and religion)

- Previous health state and mechanical ventilation, autonomy, and advance directive

- Information (type, understanding, and feeling after communication)

- Depression (depressed and non-depressed) and prognosis

We verified that independence between the family members and patient autonomy is rejected. A significant majority of respondents who opted for total physician responsibility were family members of an autonomous patient (70\% were against and 55\% for shared decision, and $43 \%$ were for family decision, $P=.09$ ). In our opinion, the families do not want to assume the burden of responsibil- 


\section{Family Reliance on Physicians' Decisions in Life-Sustaining Treatments}

Table 2. Family Member and Patient Demographics

\begin{tabular}{|c|c|c|c|c|c|}
\hline & \multicolumn{4}{|c|}{ Family Member's Preference About Life-Saving Treatment Decisions, \% } & \multirow[b]{2}{*}{$P$} \\
\hline & $\begin{array}{l}\text { Physician } \\
\text { Decision } \\
(n=54)\end{array}$ & $\begin{array}{c}\text { Shared } \\
\text { Decision } \\
(n=55)\end{array}$ & $\begin{array}{l}\text { Family } \\
\text { Decision } \\
(n=14)\end{array}$ & $\begin{array}{l}\text { Don't Know/ } \\
\text { Didn't Answer } \\
\quad(n=3)\end{array}$ & \\
\hline \multicolumn{6}{|l|}{ Family members } \\
\hline \multicolumn{6}{|l|}{ Sex } \\
\hline Male & 19 & 29 & 14 & 33 & \\
\hline Female & 81 & 71 & 86 & 67 & .30 \\
\hline \multicolumn{6}{|l|}{ Relationship to patient } \\
\hline 1st degree (spouse, son, brother) & 80 & 85 & 86 & 100 & \\
\hline Other & 20 & 15 & 14 & 0 & \\
\hline \multicolumn{6}{|l|}{ Cohabitation with the patient } \\
\hline Yes & 50 & 33 & 43 & 33 & \\
\hline No & 50 & 67 & 57 & 67 & .18 \\
\hline \multicolumn{6}{|l|}{ Residence } \\
\hline Urban & 91 & 89 & 86 & 100 & \\
\hline Rural & 9 & 11 & 14 & 0 & .19 \\
\hline \multicolumn{6}{|l|}{ Education } \\
\hline Preschool/primary & 37 & 24 & 22 & 33 & \\
\hline 6 years of school & 20 & 27 & 21 & 33 & \\
\hline College/university & 43 & 49 & 57 & 33 & .56 \\
\hline \multicolumn{6}{|l|}{ Religion } \\
\hline Without & 6 & 18 & 14 & 0 & \\
\hline With & 94 & 82 & 86 & 100 & .13 \\
\hline \multicolumn{6}{|l|}{ Patients } \\
\hline \multicolumn{6}{|l|}{ Sex } \\
\hline Male & 19 & 29 & 14 & 33 & \\
\hline Female & 81 & 71 & 86 & 67 & \\
\hline Age, median y & 75 & 72 & 70 & 79 & .83 \\
\hline \multicolumn{6}{|l|}{ Education } \\
\hline Analfabet/primary & 61 & 51 & 79 & 67 & \\
\hline 6 years of school & 28 & 20 & 7 & 0 & \\
\hline Secondary degree/university & 11 & 29 & 14 & 33 & .56 \\
\hline
\end{tabular}

ity of decision for a patient who, prior to ICU admission, was completely independent in his activities of daily living. Somehow it is a defensive posture.

\section{Discussion}

An analysis of the univariables (age and severity index) identifies a trend in patients and diseases admitted into our respiratory ICU: they are elderly, acute-on-chronic patients with a severe exacerbation, and, in this study, without any written advance directives.

The family members we surveyed had close links with the patient; a reasonable educational level; good knowledge, in most cases, of their family situations; and some degree of depression, as expected in this situation. Thus, they were in good position to represent the patient's expectations and values (eg, moral and religious). ${ }^{21}$

The bivariable analysis points out clearly, with statistical significance, that the family members desired physician involvement in the decision-making process, either alone or shared with family member. When the situation was more serious (ie, an incompetent patient) this preference was clearer. This result is to be expected, given that the decision-making process at the end of life with an incompetent patient is a burden with which few people feel able to deal alone. Thus, given the chance, they will delegate such a difficult decision to someone (a physician) who, because of knowledge and experience, is in a better position to make the best decision.

The physician-decision preference showed no relationship with the number of days of hospitalization in the ICU. In our experience, with longer stay the family's expectations and hope for clinical improvement become more somber, and they become more reliant on the physician's words and decisions. However, the small number of cases with the cutoff limit of $\geq 14$ days may have biased the results. 


\section{Family Reliance on Physicians' Decisions in Life-Sustaining Treatments}

Table 3. Family Member Depression Scores, Satisfaction With Care Provided, and Expectation About the Patient's Prognosis

\begin{tabular}{|c|c|c|c|c|c|}
\hline & \multicolumn{4}{|c|}{ Family Member's Preference About Life-Saving Treatment Decisions, \% } & \multirow[b]{2}{*}{$P$} \\
\hline & $\begin{array}{l}\text { Physician } \\
\text { Decision } \\
(n=54)\end{array}$ & $\begin{array}{c}\text { Shared } \\
\text { Decision } \\
(n=55)\end{array}$ & $\begin{array}{c}\text { Family } \\
\text { Decision } \\
(n=14)\end{array}$ & $\begin{array}{l}\text { Don't Know/ } \\
\text { Didn't Answer } \\
\quad(n=3)\end{array}$ & \\
\hline \multicolumn{6}{|l|}{ Depression } \\
\hline Without & 29 & 33 & 50 & 67 & \\
\hline Slightly & 26 & 29 & 22 & 33 & \\
\hline Moderate & 19 & 18 & 14 & 0 & \\
\hline Serious & 26 & 20 & 14 & 0 & .52 \\
\hline \multicolumn{6}{|c|}{ Care provided in ICU } \\
\hline Very good & 83 & 73 & 79 & 100 & \\
\hline Good & 17 & 27 & 14 & 0 & \\
\hline Satisfactory & 0 & 0 & 0 & 0 & \\
\hline Bad & 0 & 0 & 7 & 0 & .83 \\
\hline \multicolumn{6}{|l|}{ Prognosis } \\
\hline Very good & 4 & 6 & 0 & 0 & \\
\hline Good & 24 & 25 & 36 & 0 & \\
\hline Satisfactory & 20 & 20 & 14 & 0 & \\
\hline $\mathrm{Bad}$ & 46 & 45 & 36 & 100 & \\
\hline Unknown & 6 & 4 & 14 & 0 & .83 \\
\hline
\end{tabular}

In this study, pre-admission autonomy, which favored the preference for physician-decision-making, seems to be the only variable influencing the decision-making process.

Since ancient Greek times the exercise of medicine in terms of physician decision-making has been based on principles of beneficence and non-maleficence. In modern medicine, however, the conduct underlying that process is structured, at least in some countries (United States and northern Europe), around the principle of autonomy (or patient/family compromise), ${ }^{22}$ whereas in southern European countries the principle of beneficence is still ascendant. In the daily exercise of medicine a confrontation exists between the perception and practice of these 2 principles: beneficence and autonomy. Of late, in the southern European medical community the principle of autonomy is slowly making inroads in the behavior of physicians. In our opinion this rise of the principle of autonomy may stem more from a defensive attitude on the part of the physician, rather than from a belief in the intrinsic competency of patients and families in free and rational reasoning. Whatever the reason, it is pertinent for physicians in southern European countries to know and understand what families think about the surrogate decision-making process in end-of-life situations, namely in life-sustaining treatments.

In looking at delegated responsibility, a consideration of values is also paramount. It is well known that it is the surrogate's responsibility to represent the personal values of the patient and no else's. However, as Ortega y Gasset, Spanish humanist and philosopher, observed, "I am I plus my circumstances." Thus, it seems utopian to believe that in such a situation the surrogate can make a decision completely independent of his own existence and the elements surrounding him, which are decisive influences over his judgment. This idea is demonstrated by the fact that in the present study patient autonomy prior to admission seemed to statistically influence decision-making preferences. The likely emotional state of family members also raises a question: can frail and anguished family members make a free and rational judgment?

Finally, the sociological and cultural components of the families must also be considered. According to Edward T Hall, an American anthropologist, culture influences the way people think, feel, and act: "Culture is communication and communication is culture." ${ }^{23}$ Therefore, it is foreseeable that different cultures would communicate in different ways. In the case of doctor-patient/family, this communication will probably not be identical.

To the best of our knowledge, this is the first and only study of its kind in Portugal. Our goals were to find out which option the surrogate would prefer regarding the responsibility of decision (physician, shared, or family decision) and if that choice would depend on the patient's competency and the influence of certain variables - the patient, the surrogate family member, and the circumstances of the situation. Our data analysis suggests that for family members of incompetent patients, the principle of autonomy can be exercised by delegating to the physician, alone or together with the family member, the responsibility of decision. Further, only one variable, autonomy prior to admission, had a statistically significant influence on that choice. Other variables need further study. A greater knowl- 


\section{Family Reliance on Physicians’ Decisions in Life-Sustaining Treatments}

edge, considering each country's cultural and social reality, is fundamental to understanding the process of decision-making to avoid or at least minimize potential ethical conflicts in ICUs.

\section{Limitations}

First, the small number of answers in some groups could be a source of bias in the analysis. Second, as in any study wherein the variables may be highly subjective, the answers must be interpreted carefully. We tried to avoid the individual error of misinterpretation by limiting to 2 the number of physicians who administered the questionnaire, the author being responsible for more than $90 \%$ of the interviews. The interviews also took place in a serene atmosphere, as we attempted to put the families at ease. We explained the goals of the study to the families, along with our expectations from their answers, to avoid any ambiguity. We also clarified that, whatever their preferences, their choices would not influence the ICU staff decisions. The family members seemed comfortable during the interviews, and many of them praised the study. Although we made efforts to put the family members at ease, it is not always possible for people to exchange their views in an open and sincere manner. Third, a variable that was not considered but that might influence the respondents' preferences is the economic impact of disease on the family. Considering the diminished health status and physical autonomy of these patients - and, in most cases, their poor economic status-their care may have been an economic burden in some families, which could have implications for their decision-making. Fourth, the extent and limits of family involvement in the decision-making process were not completely clear. These limitations and factors indicate the need for further studies of this kind in Portugal.

\section{Conclusions}

The majority of families preferred to rely on the physicians, whereas some want to share the decision-making process. In a shared decision it is important to know if they want to have a say or simply to be informed. This study also points out that factors such as autonomy can influence the family's preferences.

Finally, this study has greatly clarified one aspect: the social and cultural setting of each country-including the literacy level of family members, the way the family and the physician interact, the kind of information the physicians provide, and the extent of family involvement physicians are prepared to accept-are major factors to be considered and reflected upon in any analysis of surrogate decision-making.

\section{ACKNOWLEDGMENTS}

Thanks to Leonor Nicolau, Preventative Medicine Institute, Lisbon, Portugal, for assistance with statistics; Pilar Azevedo, MD, Santa Maria University Hospital, Lisbon, Portugal, for assistance in interviewing family members; Rosario Monteiro, TechDreams Company and Luis Santos, Regional Health Administration, Lisbon, Portugal, for assistance in informatics, and Luis Pereira da Silva, MD PhD, Dona Estefânia Hospital, Lisbon, Portugal, for reviewing the manuscript.

\section{REFERENCES}

1. Gavrin JR. Ethical considerations at the end of life in the intensive care unit. Crit Care Med 2007;35(2 Suppl):S85-S94.

2. Sprung CL, Eidelman LA. Worldwide similarities and differences in the foregoing of life-sustaining treatments. Intensive Care Med 1996; 22(10): 1003-1005.

3. Ferrand E, Bachoud-Levi AC, Rodrigues M, Maggiore S, BrunBuisson C, Lemaire F. Decision-making capacity and surrogate designation in French ICU patients. Intensive Care Med 2001;27(8): 1360-1364.

4. Brock D. What is the moral authority of family members to act as surrogate for incompetent patients? Milbank Q 1996;74(4):599-618.

5. Emanuel E, Emanuel L. Proxy decision making for incompetent patients: an ethical and empirical analysis. JAMA 1992;267(15): 2067-2071

6. Azoulay E, Pochard F, Chevret S, Adrie C, Annane D, Bleichner G, et al. Half the family members of intensive care unit patients do not want to share in the decision-making process: a study in 78 French intensive care units. Crit Care Med 2004;32(9):1832-1838.

7. Heyland DK, Cook DK, Rocker GM, Dodek PM, Kutsogiannis DJ, Peters S, et al. Decision-making in the ICU: perspectives of the substitute decision-maker. Intensive Care Med 2003;29(1): 75-82.

8. Yaguchi A, Truog RD, Curtis JR, Luce JM, Levy MM, Mélot C, Vincent JL. International differences in end-of-life attitudes in the intensive care unit: results of a survey. Arch Intern Med 2005;165(17): 1970-1975.

9. Vincent JL. Ethical principles in end-of-life decisions in different European countries. Swiss Med Wkly 2004;134(5-6):65-68.

10. Levy MM. Evaluating our end-of-life practice. Crit Care 2001;5(4): 182-3.

11. European Judicial Network in Civil and Commercial Matters. http:// ec.europa.eu/civiljustice/index_en.htm. Accessed January 6, 2014.

12. Cardoso T, Fonseca T, Pereira S, Lencastre L. Life-sustaining treatment decisions in Portuguese intensive care units: a national survey of intensive care physicians. http://ccforum.com/content/7/6/R167. Accessed January 6, 2014.

13. Damasio A. Descartes' error: emotion, reasoning, and the human brain. New York: Harper; 1995.

14. Pochard F, Azoulay E, Chevret S, Lemaire F, Hubert P, Canoui P, et al. Symptoms of anxiety and depression in family members of intensive care unit patients: ethical hypothesis regarding decisionmaking capacity. Crit Care Med 2001;29(10):1893-1897.

15. Mechanic D, Meyer S. Concept of trust among patients with serious illness. Soc Sci Med 2000;51(5):657-668.

16. Evans LR, Boyd EA, Malvar G, Apatira L, Luce JM, Lo B, White DB. Surrogate decision-makers' perspectives on discussing prognosis in the face of uncertainty. Am J Respir Crit Care Med 2009; 179(1):48-53.

17. Bisbing SB. Competency and capacity: a primer. In: Sarban SS, Gibofsky A, Firestone MH, editors. Legal medicine, 1st edition. St Louis: Mobsy;1988:32-43. 


\section{Family Reliance on Physicians' Decisions in Life-Sustaining Treatments}

18. Silver Innings Foundation. http://www.silverinnings.com. Accessed January 6, 2014.

19. Nazir IL, Timothy SW. Prolonged mechanical ventilation in critically ill patients: epidemiology, outcomes and modeling the potential cost consequences of establishing a regional weaning unit. Crit Care 2011;15(2):R102 DOI: $10.1186 / \mathrm{cc} 10117$.

20. Wakatsuki M, Sadler P. Invasive mechanical ventilation in acute exacerbation of COPD: prognostic indicators to support clinical decisions. JICS 2012;13(3):238-243.
21. Arnold RM, Kellum J. Moral justifications for surrogate decision making in the intensive care unit: Implications and limitations. Crit Care Med 2003;31(5 Suppl):S347-S353.

22. White DB, Braddock CH 3rd, Bereknyei S, Curtis JR. Towards shared decision making at the end of life in intensive care units: opportunities for improvement. Arch Intern Med 2007;167(5):461-467.

23. Rogers ME, Hart WB, Mike Y. Edward T. Hall and the history of intercultural communication: the United States and Japan. Keio Comm Rev 2002;24:3-26. 